\title{
Metastable states in magnetic nanorings
}

Castaño, F. J.; Ross, C. A.; Frandsen, Cathrine; Eilez, A.; Gill, D.; Smith, H. I.; Redjdal, M.; Humphrey, F. B.

Published in:

Physical Review B Condensed Matter

Link to article, DOI:

10.1103/PhysRevB.67.184425

Publication date:

2003

Document Version

Publisher's PDF, also known as Version of record

Link back to DTU Orbit

Citation (APA):

Castaño, F. J., Ross, C. A., Frandsen, C., Eilez, A., Gill, D., Smith, H. I., Redjdal, M., \& Humphrey, F. B. (2003). Metastable states in magnetic nanorings. Physical Review B Condensed Matter, 67(18), 184425.

https://doi.org/10.1103/PhysRevB.67.184425

\section{General rights}

Copyright and moral rights for the publications made accessible in the public portal are retained by the authors and/or other copyright owners and it is a condition of accessing publications that users recognise and abide by the legal requirements associated with these rights.

- Users may download and print one copy of any publication from the public portal for the purpose of private study or research.

- You may not further distribute the material or use it for any profit-making activity or commercial gain

- You may freely distribute the URL identifying the publication in the public portal 


\title{
Metastable states in magnetic nanorings
}

\author{
F. J. Castaño and C. A. Ross \\ Department of Materials Science and Engineering, Massachusetts Institute of Technology, Cambridge, Massachusetts 02139 \\ C. Frandsen \\ Department of Physics, Technical University of Denmark, DK-2800 Kgs.Lyngby, Denmark
}

A. Eilez, D. Gil, and Henry I. Smith

Department of Electrical Engineering and Computer Science, Massachusetts Institute of Technology, Cambridge, Massachusetts 02139

M. Redjdal and F. B. Humphrey

Department of Electrical and Computer Engineering, Boston University, Boston, Massachusetts 02215

(Received 13 February 2003; published 30 May 2003)

\begin{abstract}
Magnetization states and hysteresis behavior of small ferromagnetic rings, of diameters 180-520 nm, have been investigated using magnetic force microscopy. In addition to the expected bi-domain ("onion") and flux-closed ("vortex") magnetization states, a metastable state has been found. This "twisted" state contains a $360^{\circ}$ domain wall which can exist over a wide range of applied fields. Four possible configurations of the twisted state are possible. Micromagnetic modeling shows that the twisted state is stabilised in small diameter, narrow rings. Additionally, more complex configurations such as double twisted states with two $360^{\circ}$ walls have been observed.
\end{abstract}

DOI: $10.1103 /$ PhysRevB.67.184425

PACS number(s): 75.75.+a, 75.60.-d, 85.75.-d

\section{INTRODUCTION}

There is increasing interest in the magnetic properties of small magnetic solids (nanomagnets), with thicknesses of a few nanometers and deep-submicron lateral dimensions. ${ }^{1,2}$ These structures can be engineered to display different stable magnetized states depending on their shape, dimensions, and composition. An understanding of the stability of different states, and the ability to control the switching field, enables nanomagnets to find application in high-density magnetic random access memories, ${ }^{2}$ magnetic logic, ${ }^{3}$ or other magnetoelectronic devices. The majority of work has been carried out on magnetic discs, wires, or bars, but ring-shaped magnets have recently been the subject of several theoretical ${ }^{4}$ and experimental ${ }^{5-8}$ studies. The ring shape is particularly interesting because of the existence of flux-closed magnetic configurations ("vortex" states), in which the magnetization runs circumferentially around the ring, either clockwise or counterclockwise, which may make rings ideal candidates for high-density storage devices. ${ }^{4}$ The transitions between the magnetic states in rings can give information about the nucleation, movement and annihilation of domain walls in well-controlled structures.

To date, micron-size rings ${ }^{5,6}$ and $300-800 \mathrm{~nm}$ wide octagonal ring structures ${ }^{7,8}$ have been produced. The experimental results support the existence of just two different magnetic states: one being the flux-closure or "vortex" state and the other a state with two domain walls, known as an "onion" state. However, the behavior of submicron circular rings has not been investigated, and fundamental questions remain, in particular which magnetic states are stable, and the mechanism by which the ring switches from one state to another. We have fabricated circular rings with diameters of $180 \mathrm{~nm}$ and above, and widths of $30 \mathrm{~nm}$ and above, and have characterized and modeled their magnetic behavior. In the present paper we present clear experimental evidence for the existence of additional magnetic states in submicron ring-shaped ferromagnets, and discuss the evolution of the magnetic state of small rings as a function of applied field.

\section{EXPERIMENTAL METHODS}

Nanorings were fabricated by a liftoff process from ringshaped patterns written into a resist layer by electron-beam lithography. To create shapes as close as possible to a perfect ring, the electron beam was deflected in a circular trajectory with a precision of $2 \mathrm{~nm}$. Circles were made using singlepixel lines spaced $18 \mathrm{~nm}$. By varying the number of singlepixel lines and the $e$-beam dose, rings with submicron dimensions were produced. The nanorings had outer diameters of 180-520 nm and widths of 30-200 nm. Magnetic films of Co or permalloy $\left(\mathrm{Ni}_{80} \mathrm{Fe}_{20}\right)$ with thicknesses of $10 \mathrm{~nm}$ were deposited onto the resist patterns. To prevent oxidation, magnetic films were coated with $3 \mathrm{~nm}$ of $\mathrm{Cu}$ or Au. The films were made either by electron-beam evaporation in a chamber with a base pressure of $10^{-6}$ Torr, or by ion-beam sputtering at 0.1 mTorr in a chamber with a base pressure of $10^{-9}$ Torr. In each case the films were polycrystalline with grain size of approximately $10 \mathrm{~nm}$, and substrates were oxidized silicon. As an example, Fig. 1 shows arrays of nanorings with varying dimensions.

The magnetic states of the nanorings were imaged by magnetic force microscopy (MFM), using a Digital Instruments Nanoscope with a low-moment commercial tip. The tip height during scanning was typically $35 \mathrm{~nm}$. The nanorings were saturated in an in-plane magnetic field of $1 \mathrm{~T}$, then the field was removed and the rings were imaged at rema- 

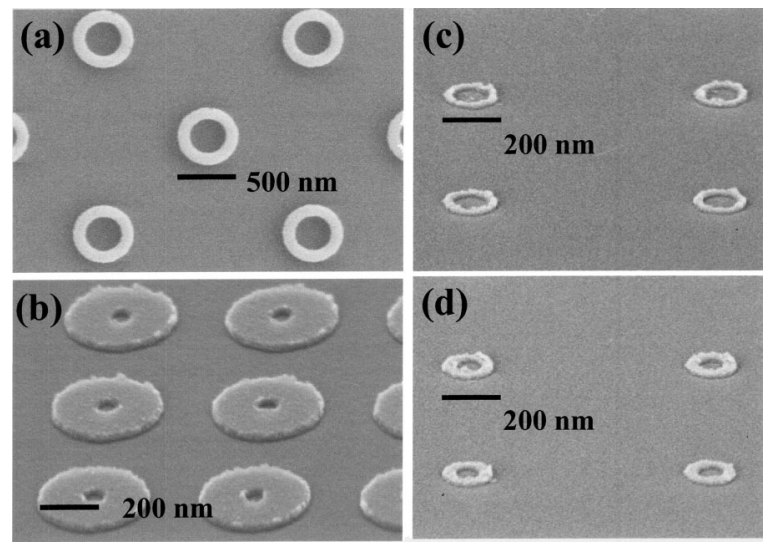

FIG. 1. Plan-view and tilted scanning electron micrographs of four arrays of Co rings with diameters and widths of (a) 520 and $120 \mathrm{~nm}$, (b) 360 and $160 \mathrm{~nm}$, (c) 190 and $30 \mathrm{~nm}$, and (d) 180 and 50 $\mathrm{nm}$.

nence. Further images were recorded after applying then removing reverse fields of various amplitudes, using permanent magnets attached to an adjustable fixture.

\section{RESULTS AND DISCUSSION}

Figures 2(a)-2(f) show the evolution of the magnetic states of one 520-nm diameter Co ring as a function of reverse field. After saturation, the ring is present in an onion state (shown schematically in the top panel of Fig. 2), which is characterized by dark and light contrast at opposite sides of the ring originating from the two domain walls. At a certain reverse field, in this case 299 Oe, the ring "disappears" from the image as a vortex state forms, Fig. 2(e). The vortex has zero external field so it does not produce any MFM contrast. Both the onion and vortex states have been identified previously in rings. ${ }^{5,7}$ However, over a range of fields smaller than that needed to produce the vortex state, a state is visible which we call a twisted state. This state, which can be seen in Figs. 2(c) and 2(d), is characterized by adjacent light and dark contrast at one side of the ring. There are four possible variants of the twisted state, depending whether the dark-light contrast is on the left or right of the ring, and whether the dark spot is above or below the light spot. All of these variants have been observed in our arrays. This behavior contrasts with that seen in larger rings where a direct transition from an onion to a vortex was reported. ${ }^{5}$ Although Fig. 2 shows a $520 \mathrm{~nm}$ diameter ring, similar magnetization states can be seen in 180 and $360 \mathrm{~nm}$ rings, though the images have less contrast due to the lower moment of the rings.

From the MFM, it appears that the twisted state is formed from the onion state by the movement of one wall around the ring until it reaches the other wall. Significantly, the resulting configuration can have an extensive range of stability with respect to applied field. Although common, the twisted state is not seen in all rings in an array. For instance, out of 64 rings similar to that of Fig. 2(a), 15 showed twisted states. Figure $2(\mathrm{~g})$ shows the stability ranges for the twisted states seen in these 15 rings: in one ring the twisted state existed for applied fields between 60 and $460 \mathrm{Oe}$. The twisted states
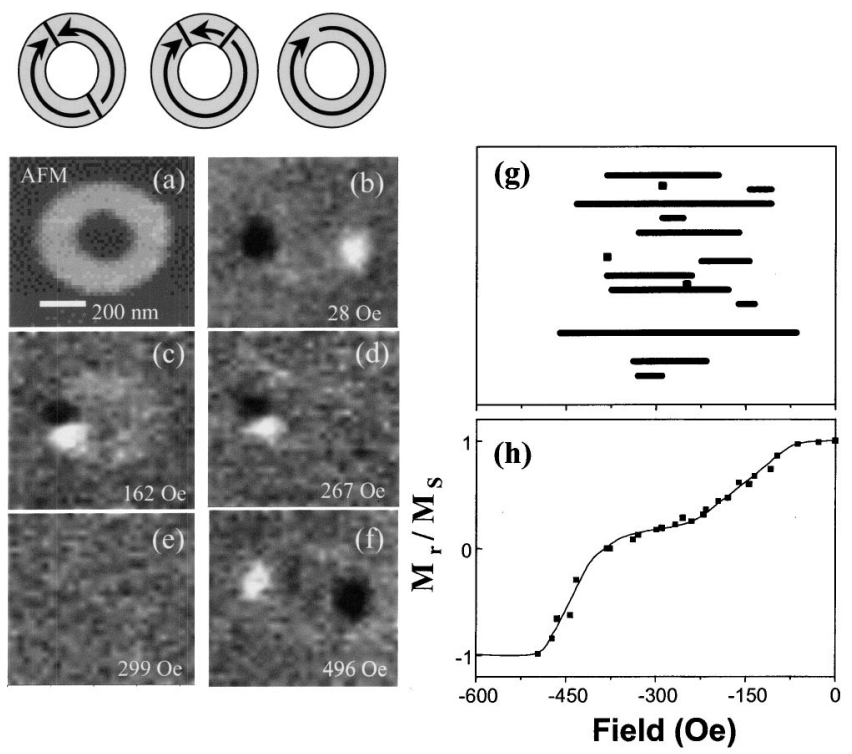

FIG. 2. At the top, a schematic of the onion, twisted and vortex states in a ring. Below, data from a $520 \mathrm{~nm}$ diameter ring: (a) an atomic force micrograph. (b)-(f) a sequence of MFM images measured at remanence after first saturating the sample at $10000 \mathrm{Oe}$, then applying and removing a reverse field of (b) $28 \mathrm{Oe}$, (c) $162 \mathrm{Oe}$, (d) $267 \mathrm{Oe}$, (e) $299 \mathrm{Oe}$, and (f) $496 \mathrm{Oe}$. (g) a plot showing the range of stability of the twisted state for 15 rings out of a 64-ring array, for rings of diameter $520 \mathrm{~nm}$ and width $175 \mathrm{~nm}$. Each of the horizontal bars represents the field range over which the twisted state was seen in an individual ring; there is a wide range of behavior. (h) Remanent hysteresis loop of the 64-ring array. The low-field step, corresponding to the formation of twisted or vortex states, is smoothed out by the variability in switching field between individual rings.

do not represent a configuration where two domain walls are coincidentally pinned next to one another by an irregularity in the ring. If that were the case, we might expect to see other examples where walls are pinned at different positions around the rings. All of our twisted states have the same MFM contrast, so appear to represent a distinct magnetic state containing two interacting walls that do not annihilate each other. At sufficiently high fields, the twisted states turn into vortex states and eventually into onion states of opposite orientation to the starting state, as shown in Fig. 2(f).

Within the arrays, there is a range of behavior, evident from Fig. 2(g). The transitions between onion, twisted, and vortex states occur at different fields for individual rings. Some rings do not show twisted states, indicating a direct transition from the onion to the vortex state or even from the onion to the reverse onion state ${ }^{5}$ another possibility is that the stability range of the twisted state in these rings is smaller than the field intervals used for imaging. Trends in behavior can be followed by plotting collective hysteresis loops for arrays of 64 rings, based on the MFM images. If the original saturating field is applied in the $+x$ in-plane direction, then a moment of \pm 1 is assigned to rings showing an onion state oriented along $\pm x$, and 0 for a vortex or twisted state. Figures 2(h) and 3 show hysteresis loops for arrays of rings, $520 \mathrm{~nm}$ diameter and $360 \mathrm{~nm}$ diameter, re- 


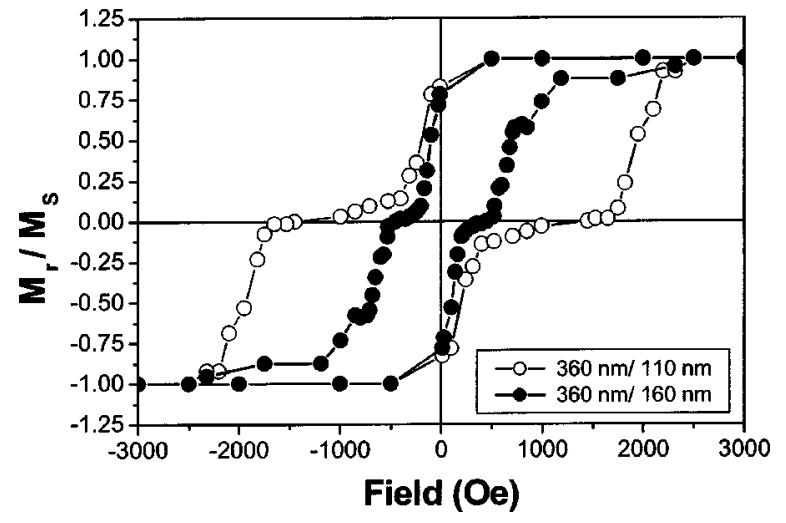

FIG. 3. Remanent hysteresis loops calculated from MFM data for two arrays with ring diameters of $360 \mathrm{~nm}$ and widths of 110 and $160 \mathrm{~nm}$, similar to Fig. 1(b). The steps showing the destruction of the initial onion state and the formation of the reverse onion state (at higher field) are clear.

spectively. Two steps can be seen in the loops, as reported for larger rings: $5,7,9$ a low-field step corresponding to the destruction of the positive onion state, and a high-field step corresponding to the creation of the reverse onion state. Between these steps is a plateau region corresponding to the existence of vortex or twisted states. The steps occur at higher fields for rings with narrower widths.

The differences in response of individual rings are attributed to edge roughness or microstructural variation, which can locally alter the nucleation or pinning fields of domain walls. ${ }^{5,10,11}$ For instance, notches are known to pin walls in rings. ${ }^{12}$ An edge roughness of a few $\mathrm{nm}$ is inevitable during lithographic processing, and the random orientation of grains within the films also causes local variations in magnetocrystalline anisotropy. These small-scale fluctuations make it likely that one wall will begin to move at a lower applied field than the other, leading to the formation of a twisted or vortex state, instead of a direct transition from one onion state to the other by the simultaneous movement of the two walls. ${ }^{5}$ It is worth pointing out that since the twisted states have almost zero remanence, magnetometry methods would be incapable of distinguishing between vortex and twisted states. In contrast to $\mathrm{Co}$ rings, $\mathrm{NiFe}$ showed much smaller switching fields, and the magnetic states were strongly perturbed by the low-moment tip, preventing quantitative measurements of switching fields.

To characterize the twisted state it is necessary to consider the nature of the domain walls in narrow thin-film structures. In the onion state, two head-on domain walls are present in each nanoring. Such head-on walls can have different magnetic configurations depending on the width and thickness of the magnetic strip. For narrow, thin rectangular strips, the wall has a transverse (Néel) character in which all moments lie in plane, while head-on walls in wider strips have a fluxclosure structure containing an out-of-plane vortex. ${ }^{13}$ We used a $3 \mathrm{D}$ micromagnetic model ${ }^{14}$ to calculate equilibrium magnetization states in magnetic rings, neglecting magnetocrystalline anisotropy. Rings are discretized into cubic cells typically $2 \mathrm{~nm}$ across, and the Landau-Lifschitz-Gilbert equation is solved for the magnetization in each cell using

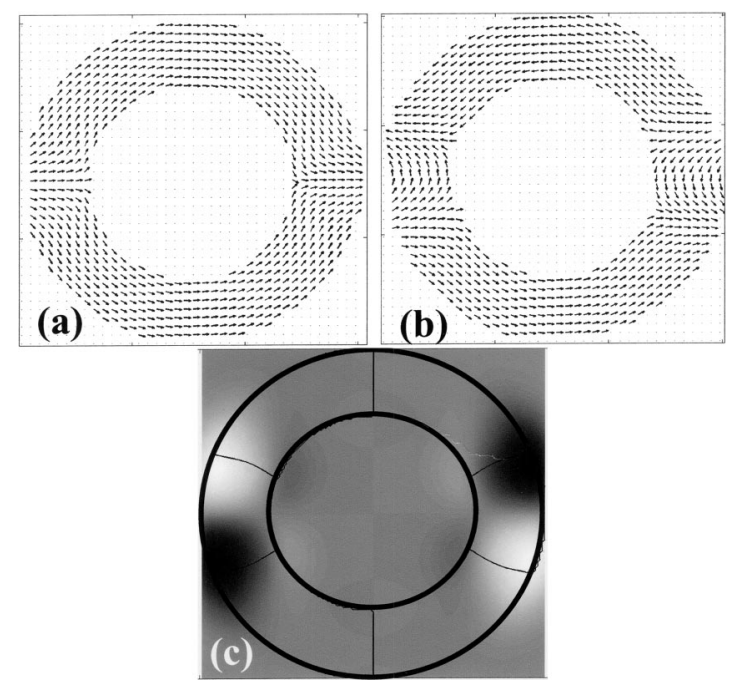

FIG. 4. Micromagnetic simulations of magnetization patterns in $150 \mathrm{~nm}$ diameter, $30 \mathrm{~nm}$ wide, and $10 \mathrm{~nm}$ thick rings. (a) Onion state obtained by saturating the magnetization in plane, then relaxing at zero field. (b) A state containing two $360^{\circ}$ walls, at remanence. (c) Greyscale image of the $z$ component of the field above the configuration in (b), at a height of $35 \mathrm{~nm}$. The maximum field is \pm 600 Oe.

the saturation moment for Co, an exchange constant of $10^{-6} \mathrm{erg} \mathrm{cm}^{-1}$, a gyromagnetic ratio of $0.0179 \mathrm{Oe} \mathrm{s}^{-1}$ and a damping constant of 1 to obtain rapid convergence. For narrow Co rings we found that the head-on walls have a transverse character, in agreement with other results on rings ${ }^{6,7,15,16}$ and rectangular strips. ${ }^{13}$ As an example, Fig. 4(a) shows a remanent onion-state obtained by equilibration after in-plane saturation.

The transition from the onion state to the vortex state has been studied previously using micromagnetics. If one wall is pinned by a notch or other asymmetry in the ring then a vortex state forms by the movement of the unpinned wall until it annihilates the pinned wall. ${ }^{5,15-17}$ However, our modeling showed that instead of the walls annihilating, it is also possible for a stable configuration to form which is analogous to a $360^{\circ}$ head-on wall which has been described in narrow thin-film strips. ${ }^{14,18,19}$ In this structure, the magnetization rotates through $360^{\circ}$ about an axis perpendicular to the film plane. We were able to model this structure by artificially placing four transverse domain walls in a ring and allowing the structure to equilibrate. This leads to a configuration which contains two pairs of walls, as shown in Fig. 4(b). Each pair forms a complete $360^{\circ}$ wall with in-plane moments. Of course, the $360^{\circ}$ walls are metastable states, having a higher energy (both exchange and magnetostatic) than a vortex state, but the $360^{\circ}$ wall in the model represents a local energy minimum and cannot relax into a vortex state unless it is perturbed. Such structures have been modeled ${ }^{18}$ and observed by electron microscopy ${ }^{19}$ in rectangular thin film strips, but not previously in rings. Notably, our modeling showed that the walls in wider rings have a flux-closure structure, and annihilate more easily to produce a vortex state in the ring. Larger rings are therefore less likely to support twisted states. 
The stability of the $360^{\circ}$ wall in a ring may be understood from the micromagnetic simulation. Each transverse wall causes surface charge at the inner and outer edges of the ring. There is an attraction between the two transverse walls because they have opposite senses of rotation. The tendency of the walls to attract is balanced by the exchange energy in the region between the two walls, leading to an equilibrium spacing between the walls. It is interesting to compare the magnetization of a ring to the magnetization of a domain wall surrounding a cylindrical bubble in a perpendicularly magnetized film. ${ }^{19,20-22}$ Although the magnetic structure is very different, there is a topological analogy between transverse walls in small rings and Bloch lines in bubble walls. Bubble walls, similar to rings, can also support a variety of magnetization states.

To illustrate the correspondence between the $360^{\circ}$ wall and the twisted state, the out-of-plane component of the field above the ring can be compared to the MFM image. ${ }^{23}$ Individual transverse walls have stray-fields resembling an asymmetrical, radially oriented in-plane dipole, but at a height of a few times the film thickness, the details of the field distribution become blurred and the wall is imaged as a single bright or dark spot displaced towards the outer edge of the ring. ${ }^{6}$ The vertical component of the external field of the $360^{\circ}$ wall of Fig. 4(b) was calculated at a height of $35 \mathrm{~nm}$ above the ring, and is shown in Fig. 4(c), in which it appears as adjacent light and dark contrast. There is a good correspondence between the calculated field distribution of one of these $360^{\circ}$ walls and the MFM images of the twisted states shown in Figs. 2(c) and 2(d).

More complex wall configurations such as a double twisted state (Fig. 5) have also been observed in nanorings. Figure 5(a) shows a $520 \mathrm{~nm}$ diameter ring that was originally saturated to form an onion state. On application of a reverse field, an additional pair of walls nucleates within the top half of the ring, reversing the magnetization of the top half to create a vortex state containing two $360^{\circ}$ walls, shown in Figs. 5(b), 5(c). At a higher field, Fig. 5(d), the $360^{\circ}$ wall on the right side of the ring separates into two transverse walls, and one of the transverse walls moves around the lower part of the ring to reverse the magnetization direction of that half of the ring. Finally, the complex $540^{\circ}$ wall on the left collapses into a single transverse wall, producing the reverse onion state, Fig. 5(e). The same behavior was seen in repeated applied field scans. Figures 5(b) and (c) have a strong resemblance to the field distributions shown in Fig. 4(c) for a ring containing a pair of $360^{\circ}$ walls.

The existence of a twisted state in nanorings has interesting consequences for the design of magnetoelectronic devices. It increases the possible number of observable states in a single-layer nanoring from the four already identified (two opposite onion states and two opposite vortex states) to at least eight (including four variants of the twisted state). For multilayer nanorings such as spin valves or tunnel junctions, there is clearly an even larger number of possible magnetic states, with as-yet unexplored electronic or magnetotransport properties. The properties of such rings are likely to be very interesting, for example, quantum interference effects have been reported in $500 \mathrm{~nm}$ diameter rings, ${ }^{24}$ and head-on walls
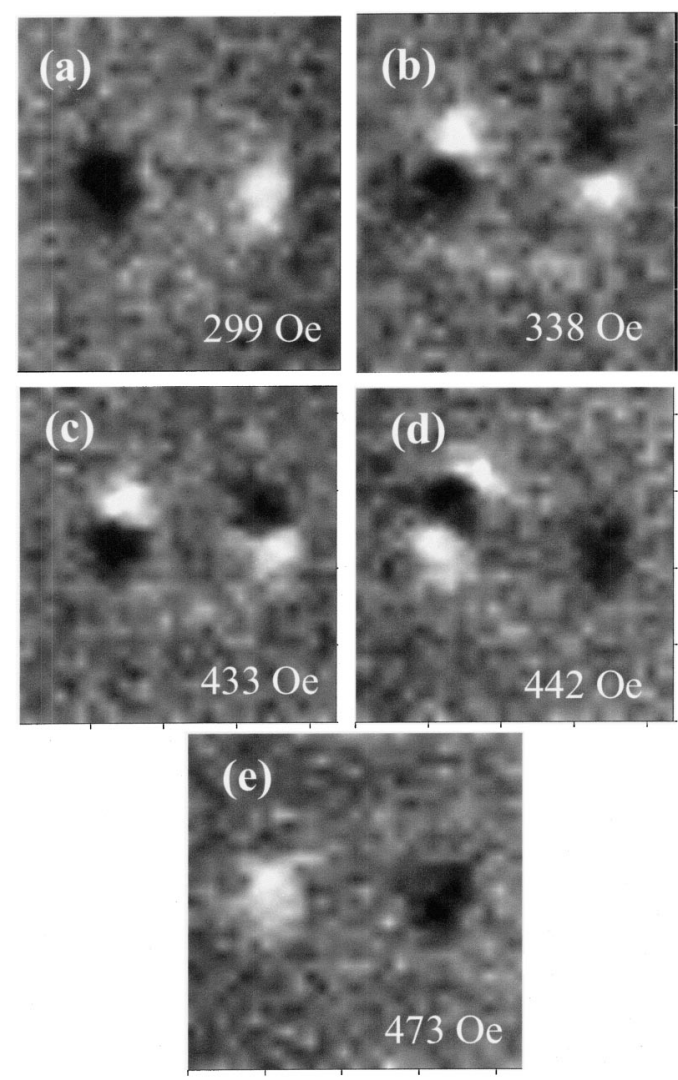

FIG. 5. Complex twisted states formed in a $520 \mathrm{~nm}$ diameter ring, imaged at remanence. (a) Onion state, following saturation then application of a reverse field of 299 Oe. (b) After applying a reverse field of (b) $338 \mathrm{Oe}$ and (c) $433 \mathrm{Oe}$, a vortex state with two $360^{\circ}$ walls is observed. (d) After applying a reverse field of $442 \mathrm{Oe}$, the right-hand $360^{\circ}$ wall separates and one transverse wall moves around the lower half of the ring. (e) The left-hand wall collapses to form a reverse onion state at 473 Oe.

in micron-size rings show measurable anisotropic magnetoresistance. ${ }^{12}$ The $360^{\circ}$ walls in twisted states may be expected to have larger magnetoresistance than a head-on wall, and could be useful in a data storage or magnetic logic device. As mentioned above, the twisted state is a higher energy configuration than a vortex state, so it represents a metastable configuration. However, we have shown that the stability range of twisted states can be several hundred Oe, exceeding the stability range over which vortex states exist, so it should be possible to create and manipulate twisted states in narrow rings or other useful geometries. The intriguing question is whether the twisted states can be sufficiently controllable to enable them to be used in data storage or logic applications, which will depend on control of the pinning and annihilation of domain walls in nanorings.

\section{ACKNOWLEDGMENTS}

The authors acknowledge the financial support of the Cambridge-MIT Institute and the Danish Technical Research Council, and Xiaobin Zhu for helpful discussion. 
${ }^{1}$ C. Stamm, F. Marty, A. Vaterlaus, V. Weich, S. Egger, U. Maier, U. Ramsperger, H. Fuhrmann, and D. Pescia, Science 282, 449 (1998)

${ }^{2}$ S. A. Wolf, D. D. Awschalom, R. A. Buhrman, J. M. Daughton, S. von Molnár, M. L. Roukes, A. Y. Chtchelkanova, and D. M. Treger, Science 294, 1488 (2001).

${ }^{3}$ R. P. Cowburn and M. E. Welland, Science 287, 1466 (2000).

${ }^{4}$ J. G. Zhu, Y. Zheng, and G. A. Prinz, J. Appl. Phys. 87, 6668 (2000).

${ }^{5}$ J. Rothman, M. Klaui, L. Lopez-Diaz, C. A. F. Vaz, A. Bleloch, J. A. C. Bland, Z. Cui, and R. Speaks, Phys. Rev. Lett. 86, 1098 (2001).

${ }^{6}$ X. Zhu, Ph.D. thesis, McGill University, Canada, 2002.

${ }^{7}$ S. P. Li, D. Peyrade, M. Natali, A. Lebib, Y. Chen, U. Ebels, L. D. Buda, and K. Ounadjela, Phys. Rev. Lett. 86, 1102 (2001).

${ }^{8}$ Y. Chen, A. Lebib, S. P. Li, M. Natali, D. Peyrande, and E. Cambril, Microelectron. Eng. 57, 405 (2001).

${ }^{9}$ M. Klaui, J. Rothman, L. Lopez-Diaz, C. A. F. Vaz, and J. A. C. Bland, Appl. Phys. Lett. 78, 3268 (2001).

${ }^{10}$ C. A. Ross, M. Hwang, M. Shima, J. Y. Cheng, M. Farhoud, T. A. Savas, Henry I. Smith, W. Schwarzacher, F. M. Ross, F. B. Humphrey, and M. Redjdal, Phys. Rev. B 65, 144417 (2002).

${ }^{11}$ J. Yu, U. Rudiger, L. Thomas, S. S. P. Parkin, and A. D. Kent, J. Appl. Phys. 85, 5501 (1999).
${ }^{12}$ M. Klaui, C. A. F. Vaz, J. A. C. Bland, W. Wernsdorfer, G. Faini, and E. Cambril, Appl. Phys. Lett. 81, 108 (2002).

${ }^{13}$ R. D. McMichael and M. J. Donahue, IEEE Trans. Magn. 33, 4167 (1997).

${ }^{14}$ M. Redjdal, P. W. Gross, A. Kazmi, and F. B. Humphrey, J. Appl. Phys. 85, 6193 (1999).

${ }^{15}$ L. Lopez-Diaz, J. Rothman, M. Klaui, and J. A. C. Bland, IEEE Trans. Magn. 36, 3155 (2001).

${ }^{16}$ L. Lopez-Diaz, J. Rothman, M. Klaui, and J. A. C. Bland, J. Appl. Phys. 89, 7579 (2001).

${ }^{17}$ L. Lopez-Diaz, M. Klaui, J. Rothman, and J. A. C. Bland, Physica B 306, 211 (2001).

${ }^{18}$ Y. Zheng and J.-G. Zhu, IEEE Trans. Magn. 33, 3286 (1997).

${ }^{19}$ X. Portier and A. Petford-Long, Appl. Phys. Lett. 76, 754 (2000).

${ }^{20}$ A. Hubert and R. Schaefer, Magnetic Domains (Springer-Verlag, Berlin, 1998).

${ }^{21}$ F. B. Humphrey and J. C. Wu, IEEE Trans. Magn. 21, 1762 (1985).

${ }^{22}$ A. H. Eschenfelder, Magnetic Bubble Technology (Springer, Berlin, 1981).

${ }^{23}$ R. Wiesendanger, Scanning Probe Microscopy and Spectroscopy (Cambridge University Press, Cambridge, UK, 1994).

${ }^{24}$ S. Kasai, T. Niiyama, E. Saitoh, and M. Miyajima, J. Appl. Phys. 91, 6938 (2002). 